CrossMark \&click for updates

Cite this: J. Mater. Chem. C, 2017, 5, 1803

Received 29th November 2016, Accepted 13th January 2017

DOI: $10.1039 / c 6 t c 05169 c$

rsc.li/materials-c

\title{
Vapor phase solvatochromic responses of polydiacetylene embedded matrix polymers $\uparrow$
}

\author{
Meng-Che Tu, ${ }^{\text {ab }}$ Jamal Ahmed Cheema, ${ }^{\text {ab }}$ Umit Hakan Yildiz, ${ }^{c}$ \\ Alagappan Palaniappan*ab and Bo Liedberg*abd
}

\begin{abstract}
The solvatochromic response of polydiacetylene (PDA) in the vapor phase is enabled upon incorporation with matrix polymers such as polyvinylpyrrolidone (PVP), polyethylene glycol (PEG), polyacrylic acid (PAA), and poly-4-vinylpyridine (P4VP). The matrix polymers provide a soft/gel-like framework for accommodating photopolymerized PDA, while facilitating its conformational alternations upon interaction with preconcentrated volatile organic compounds (VOCs). The matrix polymers enabled the differentiation of VOCs owing to their varying morphology, chemical affinity and solubility in VOCs. The ratios between PDA and the matrix polymers are optimized according to the obtained solvatochromic responses evaluated in varying temperature, humidity and storage conditions. As a proof of concept, a finger-print array for differentiation of 7 VOCs is demonstrated using matrix polymer-embedded PDA. The obtained results indicate that the response time and sensitivity of the proposed methodology supersedes previous reports on solvatochromic VOC assays. Furthermore, the proposed methodology would enable differentiation of a wide range of VOCs upon incorporation of additional matrix polymers with varying sorption properties.
\end{abstract}

\section{Introduction}

Volatile organic compound assaying is vital for the environment as well as health safety. ${ }^{1}$ Development of sensitive and selective VOC assays is therefore holding promise to avoid long term exposure to hazardous VOCs. Among various VOC assay methodologies reported, electrical and optical based transduction have attracted significant research interest due to their excellent sensing performances. ${ }^{2-8}$ While comparable sensitivities of electrical and optical systems have been reported, the latter offers several advantages such as cost effectiveness, and ease of operation and fabrication. ${ }^{9}$

Conjugated polymers such as polydiacetylene (PDA) have been explored for solvatochromic VOC assay due to its alternating structure and unique optical properties. PDA exhibits color change from blue to red upon conformational alternations in its backbone induced by external stimuli, such as $\mathrm{pH}$ change, temperature, sorption, ligand-receptor interaction, and mechanical stress. ${ }^{10-17}$ Color transition relies on the switching between

\footnotetext{
${ }^{a}$ Center for Biomimetic Sensor Science, Nanyang Technological University, Singapore 637553, Singapore. E-mail: bliedberg@ntu.edu.sg

${ }^{b}$ School of Materials Science and Engineering, Nanyang Technological University, Singapore 639798, Singapore

${ }^{c}$ Department of Chemistry, Izmir Institute of Technology, Izmir, Turkey

${ }^{d}$ Nanyang Institute of Technology in Health and Medicine, Interdisciplinary

Graduate School, Nanyang Technological University, Singapore 637553, Singapore

$\dagger$ Electronic supplementary information (ESI) available. See DOI: 10.1039/c6tc05169c
}

planar-nonplanar conformations of the backbone that influences the HOMO and LUMO levels of PDA. ${ }^{18,19}$ The change in color is usually accompanied by a change in fluorescence intensity; the maximum absorption wavelength $\left(\lambda_{\max }\right)$ changes from $640 \mathrm{~nm}$ (blue phase) to $540 \mathrm{~nm}$ (red phase), and the emission peak is revealed at $560 \mathrm{~nm}$ (red phase). ${ }^{20,21}$ Although the color of PDA changes immediately upon addition of most solvents, their vapor phase does not induce the same effect on PDA, even under saturated conditions. Therefore, terminal groups of diacetylene (DA, monomer) were modified in order to achieve better sensitivity in the vapor phase. ${ }^{22-25}$ However, these attempts on terminal group modifications of DA involve tedious synthesis protocols. In this article, a rational strategy to improve VOC response in the vapor phase is demonstrated by incorporating matrix polymers such as polyvinylpyrrolidone (PVP), polyethylene glycol (PEG), polyacrylic acid (PAA), and poly(4-vinylpyridine) (P4VP) with DA. Matrix polymers condense the VOCs in the vapor phase, subsequently concentrating them in the vicinity of PDA, thereby enabling solvatochromic responses. These matrix polymers are chosen based on their solubility in the VOCs and miscibility with DA in organic solvents. The capability of the matrix polymers to capture VOCs yields varying solvatochromic responses. Subsequently a solvatochromic response array is developed to indicate the feasibility of differentiating VOCs in the vapor phase. Compared to previous reports on solvatochromic VOC assay, the proposed methodology is facile, rapid and more sensitive to VOCs in vapor phase. 


\section{Experimental}

\subsection{Preparation of PDA cellulose membrane}

The PDA cellulose membrane was prepared by a drop-casting method as shown in Fig. 1. Briefly, $0.625 \mathrm{mg}$ of DA monomer, 10,12-docosadiynedioic acid (purchased from Sigma-Aldrich) and matrix polymer were dissolved in ethanol or dichloromethane (depending on the solubility of matrix polymer) under stirring conditions, followed by drop-casting the obtained viscous solution onto cellulose membrane at room temperature. A white colored DA embedded membrane was obtained upon evaporation of organic solvent. For control experiments, DA embedded membranes without the matrix polymers were also prepared. The membranes are exposed to UV (UV lamp, $254 \mathrm{~nm}, 6 \mathrm{~mW}$ ) for $15 \mathrm{~s}$ for polymerization yielding a blue $\mathrm{PDA} /$ matrix polymer membrane, which was subsequently utilized for VOC vapor tests. The $6 \mathrm{~mm}$ diameter punched PDA/matrix polymer membranes were then covered by aluminum foil when not in use. The matrix polymers, polyvinylpyrrolidone (PVP, average $M_{\mathrm{w}}: 40000$ ), polyethylene glycol (PEG, average $M_{\mathrm{w}}: 8000$ ), polyacrylic acid (PAA, average $M_{\mathrm{w}}: 160000$ ), and poly-4-vinylpyridine (P4VP, average $M_{\mathrm{w}}: 60000$ ) were purchased from Sigma Aldrich and used without further purification.

\subsection{VOCs vapor sensing setup}

The VOC atmosphere was created by dropping VOC solvents in a sealed chamber. The response time is recorded from closing the chamber to the time of recording the color change. The amount of VOCs is $0.5 \mathrm{~mL}$ in a $125 \mathrm{~mL}$ chamber $(0.4 \%(\mathrm{v} / \mathrm{v}))$. The images were taken using a digital camera. The RGB values were read by "imageJ" software over $100 \times 100$ pixels (about $25 \%$ of total area).

(a)

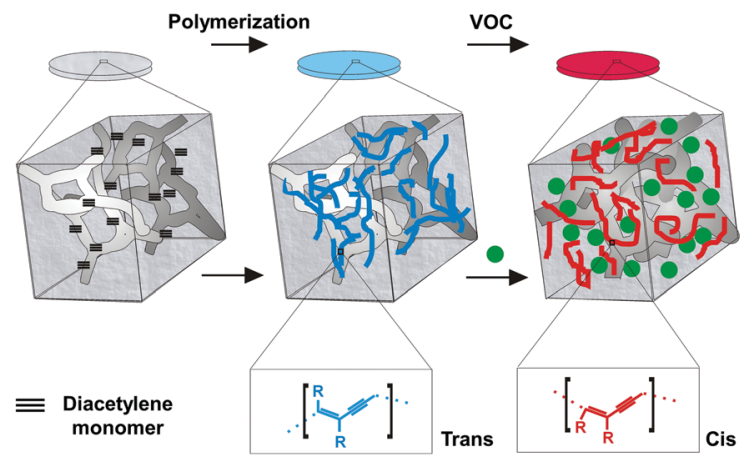

(b)

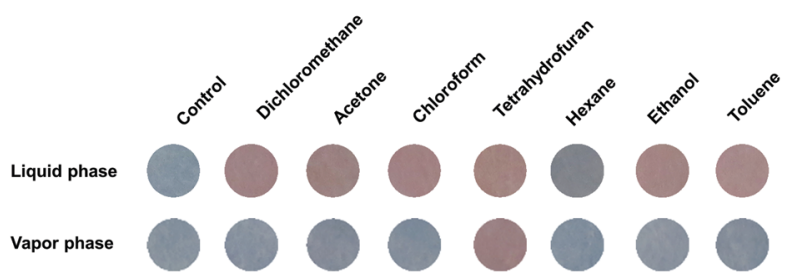

Fig. 1 (a) The mechanism of solvatochromic VOC assay using PDA/matrix polymers on a cellulose membrane. The white and grey features refers to the matrix polymers. (b) Solvatochromic responses of PDA upon exposure to $\mathrm{VOC}$ in the liquid phase and vapor phase, respectively.

\section{Results and discussion}

\subsection{Scheme and sensing mechanism}

Fig. 1a illustrates the proposed VOC assay fabrication and transduction mechanism of PDA polymer on cellulose membrane. DA monomers mixed with matrix polymers are polymerized to form PDA within the matrix polymer framework. This is evident from the observed change in color from white (DA on cellulose membrane) to blue, corresponding to PDA in a trans conformation. Upon VOC exposure, diffusion controlled swelling of the soft/gel-like matrix polymer induces mechanical distortion causing conformational change in the PDA backbone, as observed by the change in color from blue to red, corresponding to PDA in the cis conformation. For the control experiments (without matrix polymers), the color change observed upon tetrahydrofuran exposure is likely induced by distortion of PDA side chains that are associated with cellulose membranes (Fig. 1b).

Fig. 1b shows that PDA yields a rapid and distinct solvatochromic response to most VOCs in the solvent phase. However, VOCs in the vapor phase do not induce solvatochromic responses. Therefore, in order to enhance the solvatochromic response in the vapor phase, gel-like matrix polymers were utilized for providing solubilizing capability of the VOCs thereby causing mechanical distortion of PDA. The gel-like matrix exhibits swelling to varying extents upon exposure to different VOCs thereby causing deformations of the PDA backbone and unique solvatochromic responses. Various matrix polymers and PDA compositions were prepared in order to distinguish VOCs by utilizing the above mentioned phenomenon.

\subsection{Composition optimization of PDA/PVP}

Compositions of DA: matrix polymers from 1:0 to 1:400 (wt. ratio) were prepared in order to optimize the solvatochromic response of 7 VOCs in the vapor phase. Fig. 2 shows the

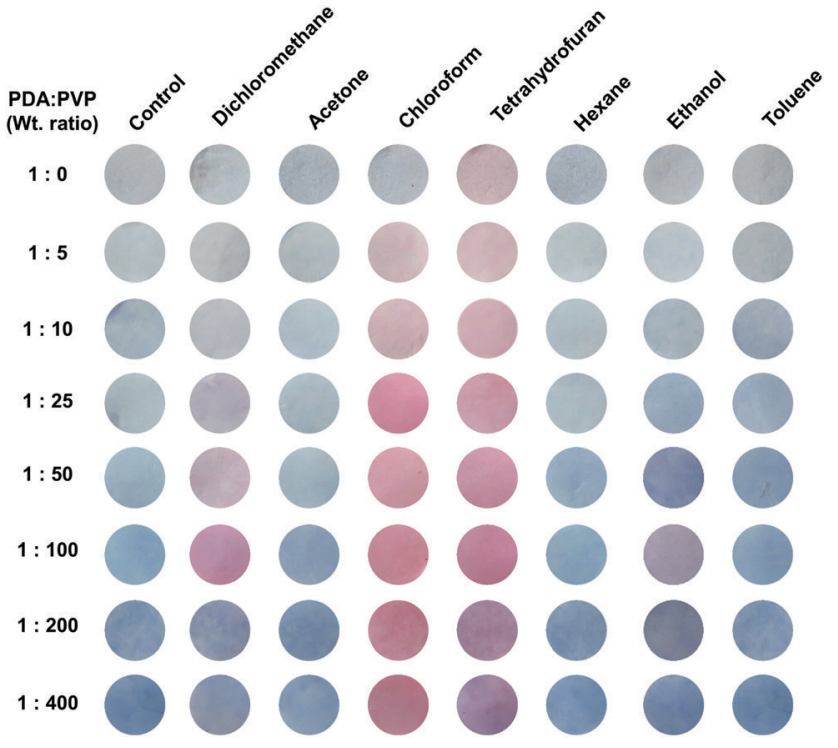

Fig. 2 The solvatochromic response of PDA embedded PVP in varying ratios upon exposure of $7 \mathrm{VOCs}$ at $0.4 \% \mathrm{v} / \mathrm{v}$ for $5 \mathrm{~min}$. 
solvatochromic response of PDA/PVP with varying weight ratios upon exposure to $0.4 \%(\mathrm{v} / \mathrm{v})$ VOC vapor. When the ratio of PDA/ PVP is 1:0 (no PVP added), only tetrahydrofuran vapor yielded evident blue-red color transition in $5 \mathrm{~min}$, indicating that only tetrahydrofuran vapor was able to alter the conformation of the PDA's backbone efficiently among the VOCs tested. Upon increasing the concentration of PVP (PDA: PVP - 1:5), a red color was observed upon exposure to chloroform vapor for $5 \mathrm{~min}$. Similar solvatochromic responses were obtained for dichloromethane and ethanol vapors at PDA:PVP ratios of $1: 25$ and $1: 50$, respectively.

In order to investigate the influence of PVP on enabling the solvatochromic response of PDA, $25 \mathrm{mg}$ PVP powder was dispersed on top of the PDA membrane and exposed to ethanol vapors. Digital images of the PVP dispersed PDA membranes recorded at an interval of $45 \mathrm{~s}$ are consolidated and shown in Fig. S1 (ESI $\dagger$ ). As observed from Fig. S1 (ESI $\dagger$ ), PVP powders dispersed on PDA appear to form a gel-like composite over time, condensing ethanol vapor thereby facilitating the change in conformation of the PDA backbone and subsequently yielding an enhanced solvatochromic response.

The overall observation is that the solvatochromic response increases with the amount of PVP. However, ratios exceeding 1:100 yielded a lower solvatochromic response. One possible explanation is that an excess amount of PVP influences the spatial arrangement of PDA in PVP, forming a thicker and denser layer of PVP surrounding PDA, thereby inhibiting VOC vapor from inducing a conformational change in the PDA backbone. The optimal ratio of PDA/PVP was therefore determined to be $1: 100$ as it yields the most evident solvatochromic response. The ratios between PDA and the other matrix polymers including PEG, PAA, and P4VP were also optimized for maximizing the solvatochromic response to the tested VOC vapors (Fig. S2-S4, ESI $\dagger$ ).

Fig. 3 demonstrates the solvatochromic sensor array with the optimized ratio of PDA/matrix polymers upon exposure to $0.4 \%(\mathrm{v} / \mathrm{v})$ VOC vapor. The matrix polymers yielded unique color patterns towards tested VOC vapors. Although PAA enabled effective photo polymerization of PDA, as observed from the dark blue color of PDA, it did not yield distinct solvatochromic response to the VOCs.

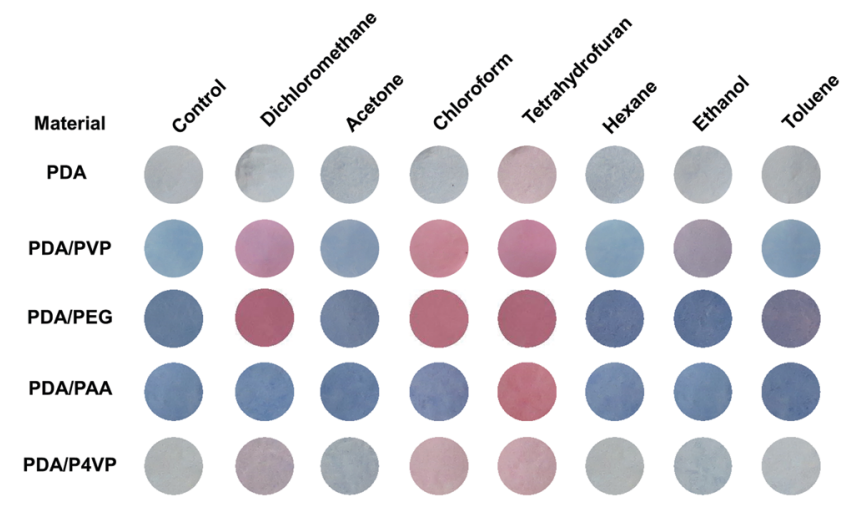

Fig. 3 The solvatochromic response of PDA embedded matrix polymers upon exposure of $7 \mathrm{VOC}$ at $0.4 \%(\mathrm{v} / \mathrm{v})$ for $5 \mathrm{~min}$; PDA : matrix polymer ratios are $1: 100$ for PDA/PVP, PDA/PEG and PDA/PAA, and $1: 10$ for PDA/P4VP.
In order to further understand the influence of matrix polymers on the solvatochromic response of PDA, polymers in powder form was used to replace the PDA/matrix polymer membrane and exposed to $0.4 \%$ VOC vapors. The weight difference of the membrane was then measured upon exposure to VOC vapors for $5 \mathrm{~min}$ (Table S1, $\mathrm{ESI} \dagger)$. The net weight increase \% was defined as $100 \% \times\left(W-W_{0}\right) /$ $W_{0}$, where $W_{0} / W$ represents the weight before/after vapor exposure, respectively. As observed from Table S1 (ESI $\dagger$ ), significant changes in weight were observed for PEG to chloroform vapor, which is almost twice the weight change observed for other VOCs. The PEG powder turned into a gel-like material, implying that the weight increase is due to the solubility of PEG in chloroform vapor. Based on this observation, it could be ascertained that the weight increase depends on VOC vapor sorption characteristics in the matrix polymer. It was observed that the solvatochromic responses of the matrix polymers/PDA (Fig. 3) correlated well to the increase in weight\% (Table S1, ESI $\dagger$ ). Furthermore, the Hildebrand solubility parameter $(\delta)$ of polymers and organic solvents (Table S2, ESI $\dagger$ ) provides a numerical estimate of the degree of interaction between them. Materials with similar values of $\delta$ indicate miscibility. The $\delta$ values of the tested VOCs ranged from 14.9 (hexane) to 26.0 (ethanol). As observed from Table S2 (ESI $\dagger$ ), the larger $\delta$ difference between hexane ( $\delta$ of 14.9) and the matrix polymers (between $\delta$ of 22.2 and 25.7) implied immiscibility, which is in agreement with the insignificant colorimetric responses, whereas smaller $\delta$ differences between the polymers and the other VOCs tested (for instance dichloromethane and chloroform) yielded significant colorimetric responses.

\subsection{Concentration dependent response of 5 PDA embedded membranes}

Solvatochromic response of all the PDA matrix polymers (PDA, PDA/PVP, PDA/PEG, PDA/PAA, and PDA/P4VP) were then evaluated for varying concentrations (v/v, $0.008 \%$ to $2 \%$ ) of 7 VOCs upon exposure for $5 \mathrm{~min}$ at room temperature (Fig. 4). The RGB values were recorded by "imageJ" software and the normalized signal was calculated by the following equation, $(R / B) /\left(R_{0} / B_{0}\right)$, where $R, B$ and $R_{0}, B_{0}$ are the values of the red and blue components of the membranes before and after VOC exposure, respectively. For the PDA embedded membrane (without polymer matrix), there is no evident color change for VOCs at concentrations lower than $0.4 \%$; solvatochromic responses of lower magnitudes were observed for $2 \%$ of dichloromethane, chloroform and toluene. As for the PDA/PAA membrane, all tested VOCs yielded indistinguishable solvatochromic responses even at high concentrations of VOC. Besides, the solvatochromic response is even lower than that of the PDA membrane for dichloromethane and toluene, which could be due to the blocking of VOC vapor from contacting the PDA embedded in the membrane by the PAA layer. PDA/PVP and PDA/P4VP membranes showed distinguishable solvatochromic responses to both dichloromethane and chloroform for concentrations above $0.4 \%$. In addition, a visible color change of the PDA/PVP membrane for ethanol vapor was observed. The addition of PEG to the PDA embedded membrane improved the solvatochromic response attributed to its ability of absorbing VOC vapors. The solvatochromic response 


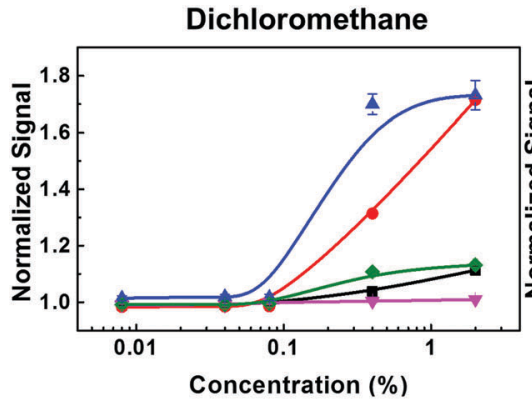

Hexane

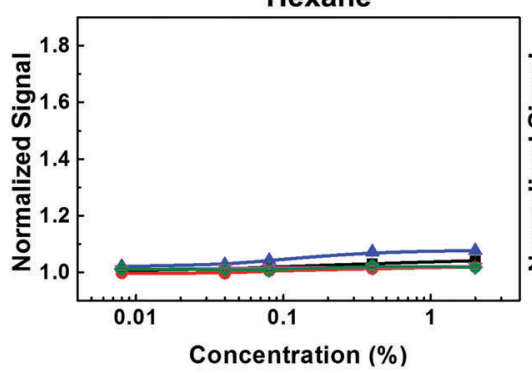

PDA

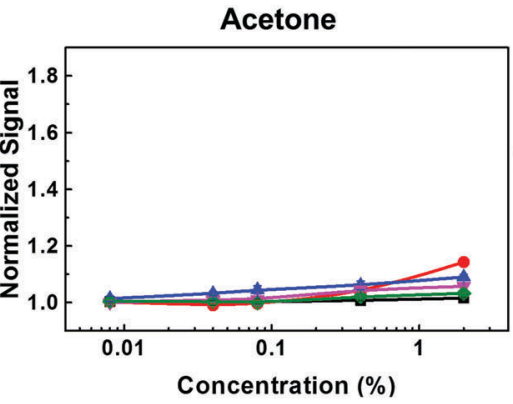

Ethanol

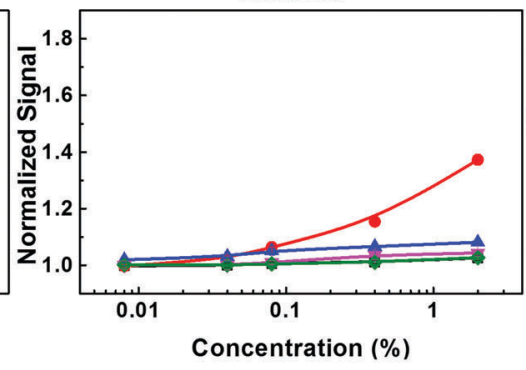

Concentration (\%)

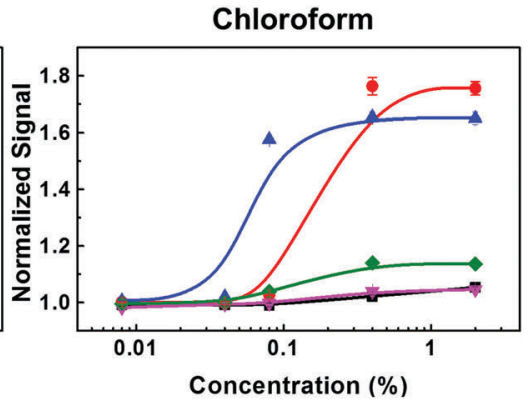

Toluene

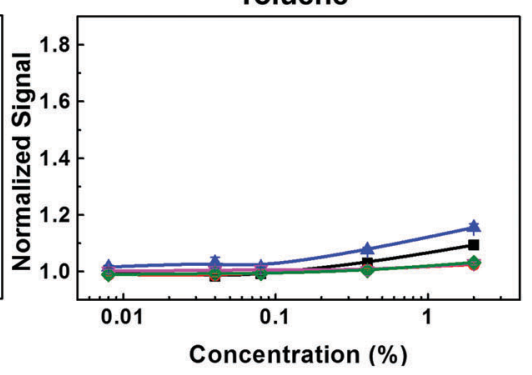

PDA/PEG

PDA/PAA

PDA/P4VP

Fig. 4 RGB analysis of 5 PDA embedded matrix polymers $(n=3)$ upon exposure to VOC vapor $(0.008-2 \%(v / v))$.

of the PDA/PEG membrane was saturated at $0.4 \%$ of dichloromethane and $0.08 \%$ chloroform vapors. Besides, the PDA/PEG membrane yielded a slightly higher solvatochromic response to toluene vapor.

As observed from Fig. 4 and Table S1 (ESI $\dagger$ ), the sorption ability of VOC vapors in the matrix polymers, for instance, sorption of chloroform in PEG yielded nearly $100 \%$ increase in weight, yielding a significant solvatochromic response. Fig. 4 shows that saturated solvatochromic responses for chloroform are obtained even at a low concentration of $0.08 \%$. On the other hand, neither PDA nor the PDA/matrix polymer responded to hexane, which could be attributed to weak interaction between hexane vapor and the matrix polymers. Furthermore, the matrix polymers which do not form a soft-gel like framework, for instance, PDA/PAA, yielded a lower solvatochromic response at a concentration of $2 \%$ dichloromethane $(\mathrm{v} / \mathrm{v})$ compared to PDA.
The obtained results therefore indicate that not every matrix polymer could facilitate color transition and that the improvement in solvatochromic response depends on the properties of the matrix polymer, primarily their solubilities in VOCs.

\subsection{Two matrix polymers mixed with the PDA embedded membrane}

The addition of a single matrix polymer to the PDA embedded membrane successfully changed the solvatochromic response upon exposure of VOC vapors. In order to further tune the solvatochromic response to VOC vapors, PDA embedded in two matrix polymers was tested for its solvatochromic response as shown in Fig. 5. The weight ratio of PDA to matrix polymers (PVP + PEG) is fixed at $1: 100$, and PVP:PEG is tested from 100:0 to $0: 100$. When there is no PEG in the membrane $(\mathrm{PDA} / \mathrm{PVP} / \mathrm{PEG}=1 / 100 / 0)$, only ethanol showed a solvatochromic response (Fig. 5a) and in cases when there is no PVP (a)

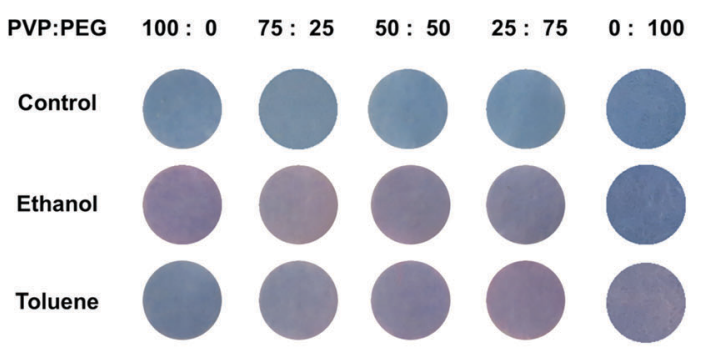

(b)

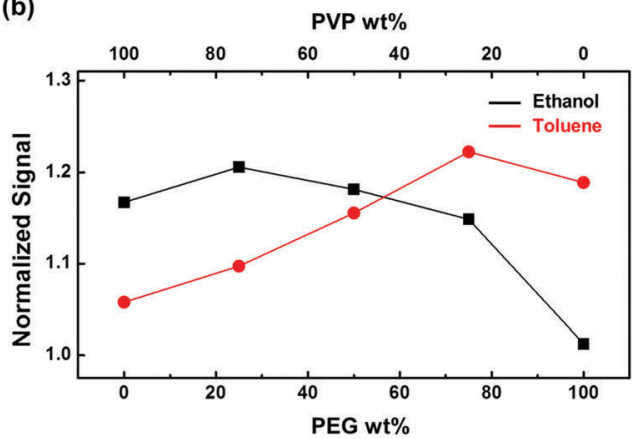

Fig. 5 (a) Solvatochromic response of PDA embedded within varying ratios of PVP and PEG, the ratio of PDA to PVP/PEG is fixed at 1 (0.625 mg). (b) RGB analysis of (a). 
(a)

\begin{tabular}{|c|c|c|c|c|c|c|c|c|c|c|c|c|c|c|c|c|c|c|c|c|c|c|c|c|c|c|c|c|c|}
\hline \multirow{2}{*}{$\begin{array}{c}\text { Vapor } \\
\text { Humidity }\end{array}$} & \multicolumn{4}{|c|}{ Dichloromethane } & \multicolumn{4}{|c|}{ Acetone } & \multicolumn{4}{|c|}{ Chloroform } & \multicolumn{4}{|c|}{ Tetrahydrofuran } & \multicolumn{4}{|c|}{ Hexane } & \multicolumn{4}{|c|}{ Ethanol } & \multicolumn{5}{|c|}{ Toluene } \\
\hline & 20 & 40 & $\begin{array}{ll}60 & 8 \\
\end{array}$ & \begin{tabular}{l|l|l}
80 & 90 \\
\end{tabular} & 20 & $\begin{array}{ll}40 & 60 \\
\end{array}$ & 80 & 90 & 20 & \begin{tabular}{l|l|}
40 & 60 \\
\end{tabular} & 80 & 90 & 20 & 40 & \begin{tabular}{l|l|}
60 & 80 \\
\end{tabular} & 90 & 20 & \begin{tabular}{l|l}
40 & 6 \\
\end{tabular} & $\begin{array}{lll}50 & 80 \\
\end{array}$ & 90 & 20 & \begin{tabular}{l|l}
40 & 6 \\
\end{tabular} & \begin{tabular}{l|l|}
60 & 80 \\
\end{tabular} & 90 & 20 & 40 & 60 & & 90 \\
\hline PDA & & & & & & & & & & & & & & & & & & & & & & & & & & & & & \\
\hline PDA/PVP & & & & & & & & & & & & & & & & & & & & & & & & & & & & & \\
\hline PDA/PEG & & & & & & & & & & & & & & & & & & & & & & & & & & & & & \\
\hline PDA/PAA & & & & & & & & & & & & & & & & & & & & & & & & & & & & & \\
\hline PDA/P4VP & & & & & & & & & & & & & & & & & & & & & & & & & & & & & \\
\hline
\end{tabular}

(b)

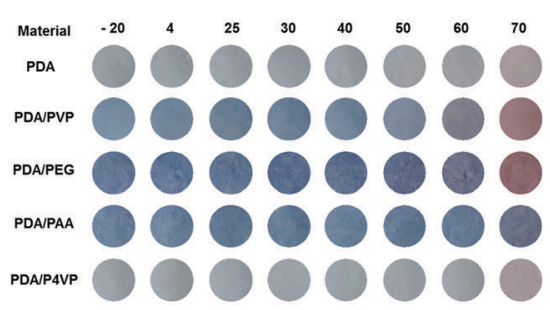

(d)

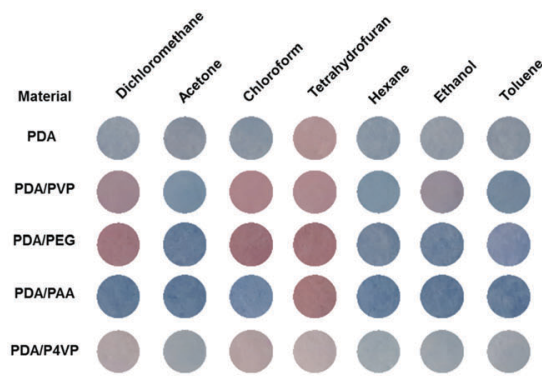

(c)

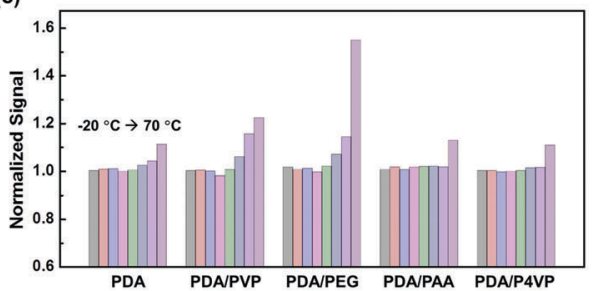

(e)

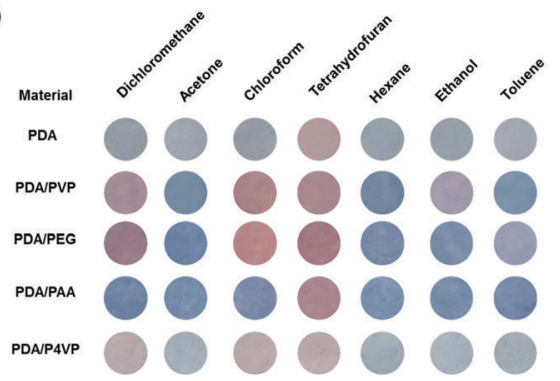

(f)

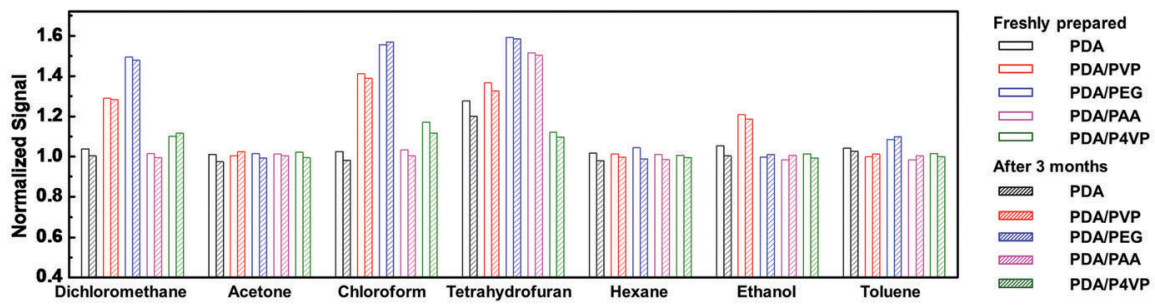

Fig. 6 (a) The solvatochromic response of matrix polymer/PDA embedded membranes under R.H. between 20\% and $90 \%$. (b) Their corresponding solvatochromic responses and (c) RGB analysis of the matrix polymer/PDA embedded membranes at varying temperatures. Solvatochromic response of (d) freshly prepared and (e) matrix polymer embedded PDA stored for 3 months and (f) normalized RGB signals of freshly prepared and matrix polymer embedded PDA stored for 3 months.

in the membrane $(\mathrm{PDA} / \mathrm{PVP} / \mathrm{PEG}=1 / 0 / 100)$, only toluene showed a solvatochromic response. Fig. 5b (RGB analysis of Fig. 5a) illustrates that the increase in $\mathrm{wt} \%$ of PVP enhanced the solvatochromic response of $\mathrm{PDA} / \mathrm{PVP}$ to ethanol. On the other hand, the increase in $w \mathrm{t} \%$ of PEG also improved the response to toluene. The obtained response further confirms that the PDA/PVP membrane is sensitive to ethanol vapor and the PDA/PEG membrane is sensitive to toluene vapor. However, intermediate PVP/PEG ratios tested yielded solvatochromic response for both ethanol and toluene vapors. Therefore, PDA embedded with two or more matrix polymers would enable tuning of the solvatochromic responses, implying the potential for the development of a solvatochromic array for selective VOC assaying.

\subsection{Influence of humidity, temperature and storage time on solvatochromic responses}

The solvatochromic responses of 5 membranes to 7 VOCs vapor were evaluated in a chamber with controlled relative humidity
(R.H.) ranging from $20 \%$ to $90 \%$ as measured by an Extech humidity meter. The R.H. was controlled by introducing $\mathrm{N}_{2}$ flow (R.H. $<70 \%$ ) or by introducing wet tissue (R.H. $>80 \%$ ) into the closed chamber. Different VOCs at $0.4 \%(\mathrm{v} / \mathrm{v})$ were introduced once the desired R.H. was reached. As shown in Fig. 6a, no significant color difference was observed in the R.H. regime, which is in an agreement with RGB analysis as shown in Fig. S5 (ESI $\dagger$ ). Although PVP, PEG, and PAA are soluble in water, no significant interferences in the solvatochromic VOC responses were observed. One possible explanation is that the vapor pressure of water is relatively lower than that of the VOC vapors tested, thereby limiting matrix polymer-gel formation by water vapor that could influence a colorimetric response.

Fig. $6 \mathrm{~b}$ and $\mathrm{c}$ show the solvatochromic response and their RGB analysis under varying temperatures. Colorimetric responses were not observed for temperatures below $40{ }^{\circ} \mathrm{C}$. PDA/PEG and PDA/ PVP, PDA and PDA/P4VP, and PDA/PAA show evident changes in color at $50{ }^{\circ} \mathrm{C}, 60{ }^{\circ} \mathrm{C}$, and $70{ }^{\circ} \mathrm{C}$, respectively. Temperature ranges 
Table 1 Literature comparison

\begin{tabular}{lllll}
\hline No. & Method & VOCs & Reported conc./volume & Assay time \\
\hline 1 & Terminal group & Tetrahydrofuran, chloroform & $2 \mathrm{~mL}$ & $1 \mathrm{~h}$ \\
2 & Terminal group & 15 VOCs & $10 \mathrm{~mL}$ & 25 \\
3 & Terminal group & 4 VOCs & $2 \mathrm{~mL}$ & 22 \\
4 & Graphene stacked PDA paper & Tetrahydrofuran, chloroform & $15 \% \mathrm{v} / \mathrm{v}$ & $1 \mathrm{~h}$ \\
5 & Matrix polymer & 7 VOCs & $100 \mu \mathrm{L}$ in $125 \mathrm{~mL}(0.08 \% \mathrm{v} / \mathrm{v})$ & $2 \mathrm{~min}$ \\
\end{tabular}

over $40{ }^{\circ} \mathrm{C}$ are therefore not suitable for VOC assaying using the proposed methodology.

In order to evaluate the long term stability of these membranes, the solvatochromic responses of the membranes stored for up to three months were compared with the freshly prepared membranes. The membranes were stored in the dark at room temperature. Fig. $6 \mathrm{~d}$ and e illustrate that comparable solvatochromic responses are achievable for membranes prepared and stored for three months prior to testing. Furthermore, RGB analysis (Fig. 6f) shows that the deviation of a normalized signal is within $2 \%$ between freshly prepared and stored membranes, indicating good stability of the PDA/matrix polymer membrane. In summary, our experimental results illustrate that the as-fabricated PDA/matrix polymer embedded membrane demonstrated good reproducibility under various humidity conditions. It also displayed good thermal and longterm storage stability.

The robust PDA embedded membranes could potentially be used for fabrication of a sensor array as illustrated in Fig. 7a and $\mathrm{b}$ for VOC discrimination. The as prepared PDA membranes attached to a flexible transparent sheet further indicates the advantages of the proposed methodology such as low-cost and ease of use. Although solvatochromic responses were obtained for lower VOC concentrations than reported before using PDA $^{13,22,24,25}$ (Table 1), sub-ppm detection sensitivities have been reported using established methodologies such as chromatography, electrochemical and optical assays. ${ }^{26-29}$ However, compared to previous reports on PDA for vapor phase VOC assays, the proposed methodology is an attractive, more sensitive and provides a simpler way to enhance the solvatochromic response of PDA film by incorporating matrix polymers with PDA. Future studies will therefore emphasize on sensitivity enhancement approaches such as optimization of stoichiometric ratios of polymer materials and their composites, extending the range of matrix polymers, and design of a sophisticated miniaturized gas sensing set-up that would concentrate VOC in the proximity of PDA. (a)

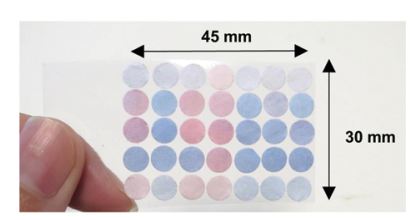

(b)

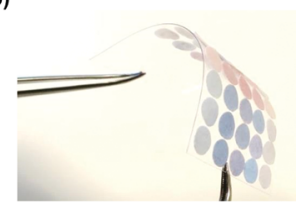

Fig. 7 (a and b) Illustration of a potential application as a sensor array on flexible substrates.

\section{Conclusions}

A facile methodology for VOC assay in the vapor phase is proposed. PDA was incorporated with matrix polymers such as PVP, PEG, PAA, and P4VP and tested for solvatochromic responses to different VOCs. PDA/matrix polymers were utilized to distinguish VOC such as chloroform, tetrahydrofuran, dichloromethane, ethanol, and toluene. The weight ratios between PDA and the matrix polymer were optimized based on the obtained solvatochromic responses. The soft gel-like matrix polymer enabled altering the conformation of the PDA backbone upon VOC exposure, subsequently yielding solvatochromic responses. Among the tested matrix polymers, PDA/ PEG demonstrated the most significant improvement in the solvatochromic response compared to the PDA embedded membrane (up to 2 orders of magnitude). Besides, mixing two matrix polymers with PDA enabled tuning the solvatochromic response, implying the possibility of the development of solvatochromic sensor arrays. Moreover, the PDA/matrix polymer membrane could be stored at room temperature in a dark environment for up to 3 months without affecting the solvatochromic response. The experimental results further illustrated that the solvatochromic responses were not influenced by R.H. variations and were amiable for operation at temperatures up to at least $40{ }^{\circ} \mathrm{C}$. Thus, the proposed methodology is a promising approach for the development of a solvatochromic sensor array for assaying a wide range of VOCs.

\section{Acknowledgements}

This work was supported by the provost office Nanyang Technological University (NTU) and MOE-Tier 1 (2014-T1-001-133-01).

\section{References}

1 B. Li, G. Sauvé, M. C. Iovu, M. Jeffries-El, R. Zhang, J. Cooper, S. Santhanam, L. Schultz, J. C. Revelli and A. G. Kusne, Nano Lett., 2006, 6, 1598-1602.

2 K. Hosono, I. Matsubara, N. Murayama, S. Woosuck and N. Izu, Chem. Mater., 2005, 17, 349-354.

3 M. C. Janzen, J. B. Ponder, D. P. Bailey, C. K. Ingison and K. S. Suslick, Anal. Chem., 2006, 78, 3591-3600.

4 J. Liu, T. Luo, F. Meng, K. Qian, Y. Wan and J. Liu, J. Phys. Chem. C, 2010, 114, 4887-4894.

5 Y. Paska, T. Stelzner, S. Christiansen and H. Haick, ACS Nano, 2011, 5, 5620-5626.

6 S. Chatterjee, M. Castro and J.-F. Feller, J. Mater. Chem. B, 2013, 1, 4563-4575. 
7 Z. Li, Q. Zhao, W. Fan and J. Zhan, Nanoscale, 2011, 3, 1646-1652.

8 T. Endo, Y. Yanagida and T. Hatsuzawa, Sens. Actuators, B, 2007, 125, 589-595.

9 U. H. Yildiz, P. Alagappan and B. Liedberg, Anal. Chem., 2012, 85, 820-824.

10 J. E. Yang, J. S. Park, E. Cho, S. Jung and S. R. Paik, Langmuir, 2015, 31, 1802-1810.

11 J. Lee, H. T. Chang, H. An, S. Ahn, J. Shim and J.-M. Kim, Nat. Commun., 2013, 4, 2461-2469.

12 I. Gill and A. Ballesteros, Angew. Chem., Int. Ed., 2003, 42, 3264-3267.

13 X. Wang, X. Sun, P. A. Hu, J. Zhang, L. Wang, W. Feng, S. Lei, B. Yang and W. Cao, Adv. Funct. Mater., 2013, 23, 6044-6050.

14 J. Lee, S. Seo and J. Kim, Adv. Funct. Mater., 2012, 22, 1632-1638.

15 Q. Cheng and R. C. Stevens, Adv. Mater., 1997, 9, 481-483.

16 J. Lee, H.-J. Kim and J. Kim, J. Am. Chem. Soc., 2008, 130, 5010-5011.

17 X. Chen, S. Kang, M. J. Kim, J. Kim, Y. S. Kim, H. Kim, B. Chi, S. J. Kim, J. Y. Lee and J. Yoon, Angew. Chem., Int. Ed., 2010, 49, 1422-1425.

18 G. Patel, R. Chance and J. Witt, J. Chem. Phys., 1979, 70, 4387-4392.
19 R. Chance, G. Patel and J. Witt, J. Chem. Phys., 1979, 71, 206-211. 20 J.-M. Kim, Y. B. Lee, D. H. Yang, J.-S. Lee, G. S. Lee and D. J. Ahn, J. Am. Chem. Soc., 2005, 127, 17580-17581.

21 J. Lee, O. Yarimaga, C. H. Lee, Y. K. Choi and J. M. Kim, Adv. Funct. Mater., 2011, 21, 1032-1039.

22 T. Eaidkong, R. Mungkarndee, C. Phollookin, G. Tumcharern, M. Sukwattanasinitt and S. Wacharasindhu, J. Mater. Chem., 2012, 22, 5970-5977.

23 B. Yoon, I. S. Park, H. Shin, H. J. Park, C. W. Lee and J. M. Kim, Macromol. Rapid Commun., 2013, 34, 731-735.

24 B. W. Davis, A. J. Burris, N. Niamnont, C. D. Hare, C.-Y. Chen, M. Sukwattanasinitt and Q. Cheng, Langmuir, 2014, 30, 9616-9622.

25 J. Yoon, S. K. Chae and J.-M. Kim, J. Am. Chem. Soc., 2007, 129, 3038-3039.

26 C. M. Drain, J. T. Hupp, K. S. Suslick, M. R. Wasielewski and X. Chen, J. Porphyrins Phthalocyanines, 2002, 6, 243-258.

27 R. Paolesse, S. Nardis, D. Monti, M. Stefanelli and C. Di Natale, Chem. Rev., 2016, DOI: 10.1021/acs.chemrev.6b00361.

28 G. Peng, U. Tisch, O. Adams, M. Hakim, N. Shehada, Y. Y. Broza, S. Billan, R. Abdah-Bortnyak, A. Kuten and H. Haick, Nat. Nanotechnol., 2009, 4, 669-673.

29 F. Röck, N. Barsan and U. Weimar, Chem. Rev., 2008, 108, 705-725. 